\title{
Lexis
}

Journal in English Lexicology

17 | 2021

Humor, creativity and lexical creation

\section{Les séries ludiques en argot et en langue familière : saut métaphorique et rebond}

Fabrice Antoine

\section{(2) OpenEdition}

1 Journals

\section{Electronic version}

URL: https://journals.openedition.org/lexis/5090

DOI: $10.4000 /$ lexis. 5090

ISSN: 1951-6215

\section{Publisher}

Université Jean Moulin - Lyon 3

\section{Electronic reference}

Fabrice Antoine, "Les séries ludiques en argot et en langue familière : saut métaphorique et rebond", Lexis [Online], 17 | 2021, Online since 15 August 2021, connection on 20 August 2021. URL: http:// journals.openedition.org/lexis/5090 ; DOI: https://doi.org/10.4000/lexis.5090

This text was automatically generated on 20 August 2021.

\section{(c) (i) (9)}

Lexis is licensed under a Creative Commons Attribution-NonCommercial-NoDerivatives 4.0 International License. 


\title{
Les séries ludiques en argot et en langue familière : saut métaphorique et rebond
}

\author{
Fabrice Antoine
}

Je souhaite dédier cet article aux plus de 650 étudiant/es de première et de deuxième années de master MéLexTra dont j'ai dirigé les travaux de mémoire ${ }^{1}$ au fil des années depuis 1995 et tout particulièrement à Camille BÉCART (1998-2021) : nous avons beaucoup appris les un/es des autres.

\section{Introduction}

1 Les argots, qui semblent toujours un champ foisonnant, voire chaotique, sont sources d'étonnement et de fascination, non pas en raison des champs sémantiques qu'ils couvrent, somme toute en nombre assez limité, mais surtout pour la façon dont s'y déploient la créativité, l'inventivité, la poésie de ceux qui en sont les créateurs en même temps que les utilisateurs.

2 Les tropes, les figures de transport de sens, en particulier la métaphore et la métonymie, y sont au service des fonctions que revêtent les argots, la fonction cryptique et la fonction conniventielle ayant longtemps primé, la fonction ludique ou même crypto-ludique (Goudaillier [1998]) étant sans doute la principale dans les argots contemporains - et c'est dans celle-là que se révèle un certain humour ; c'est du jeu avec les mots, leurs sonorités (la forme) et les images mentales qu'ils évoquent (le sens), que naît sinon un rire, du moins un sourire, sinon franc et joyeux, du moins amusé et même parfois (souvent?) teinté d'amertume ou de fatalisme, voire de cruauté. On entend bien « humour » ici au sens d'attitude de l'esprit « ne se laissant pas résumer à la production de l'hilarité » [Moura 2019:23]. C'est de la reconnaissance d'un décalage, d'un jeu (l'interstice qui permet à l'imagination de vagabonder et de juxtaposer des significations autrement non juxtaposables: ce que fait justement l'argot par pulsion cryptique puis, plus gratuitement, par pulsion ludique) que naît l'humour, qui permet de « parler de choses sérieuses, tristes, etc., de façon légère voire 
comique » [Escarpit 1960] - et dans cet « etc. », on ajoutera sans peine taboues ou crues. On reprendra la distinction de Moura [2019:27-28], pour qui l'humour est " rire avec », de façon impliquée, voire bienveillante - l'humour est partie prenante ; mais les argots rient aussi « de » et encore rient " contre ", ce qui définit pour Moura le comique et le satirique : c'est là que l'on parlera d'humour cruel, qui est détaché de son objet.

On propose d'examiner ici l'une des manières dont la pulsion ludique évoquée plus haut peut générer dans les argots des vagues de création lexicale (par opposition à la création d'un item isolé) et donc de mettre en évidence un phénomène à l'œuvre dans les argots et la langue familière, dans le cadre relativement contraint de la lexicogenèse argotique tel que je l'ai défini et étayé au fil des années et des études de champs lexicaux variés, en anglais comme en français (voir bibliographie).

\section{Des matrices lexicogéniques aux équations métaphoriques}

4 Pour rappel, contrairement à l'image que donnent des argots les dictionnaires unilingues ou bilingues (peu nombreux), d'un amas anarchique de désignations, je tente de montrer que la création lexicale en argot obéit, comme le lexique général, à un ensemble fini de règles, que les créateurs d'argot appliquent et respectent même s'ils n'en ont pas conscience. Jean Tournier [1985:51], dans Introduction descriptive à la lexicogénétique de l'anglais contemporain a défini treize matrices lexicogéniques de l'anglais, réduites ensuite à douze (Tournier [1993 : 22-24]), qu'il a ordonnées entre elles en quatre catégories, dont trois (néologie morpho-sémantique, néologie sémantique et néologie morphologique) pour les matrices internes et une pour la matrice externe qu'est l'emprunt. Ces matrices permettent de rendre compte de l'ensemble du lexique anglais.

Jean-François Sablayrolles [1996:97] a adopté le modèle de Tournier comme base de travail pour le lexique du français et lui a apporté des aménagements, dont une catégorie pragmatique, pour une matrice qu'il nomme "détournement " (voir aussi [Sablayrolles 2012]); son modèle comprend au moins 21 matrices (Sablayrolles [2003]) puisqu'il en subdivise certaines de Tournier (mais 22 in Sablayrolles [2006] et 24 in Sablayrolles [2012]), dont une externe, l'emprunt; il ajoute aux trois matrices de néologie sémantique de Tournier une nouvelle matrice intitulée " autres figures ».

Indépendamment, je me suis appuyé sur Tournier pour tenter de donner corps, en anglais comme en français, à ce que Louis-Jean Calvet [1994:35] nomme sans le décrire formellement: "les créations argotiques et populaires sont souvent le produit de "machines à créer», de "matrices sémantiques»". Il schématise, dans un développement sur les désignations de l'argent, ce qu'il appelle « matrice de base » sous la forme "argent = nourriture", ce sur quoi je me suis fondé pour définir ce que j'appelle l'équation métaphorique, à la racine d'une quantité de créations argotiques (mais pas de toutes, loin s'en faut). En examinant de nombreux mots d'argot, en anglais autant qu'en français, on y décèle souvent à la fois du sémantique et du formel ${ }^{2}$, d'autres fois uniquement l'un ou l'autre. Partant de ce constat, et trouvant curieux que Tournier mette sur le même plan le sémantique et le formel dans ses douze matrices (ce que fait aussi Sablayrolles), j'ai ébauché (voir Antoine [2001]) une machine qui soit une combinatoire de sémantique et de formel - le premier est variable: un ensemble 
hiérarchisé d'équations métaphoriques ou métonymiques dans lesquelles des sèmes différents de l'objet considéré sont haussés, mis en avant, au point que cet objet est vu comme n'étant que ce seul sème. Le formel, lui, est invariable: un ensemble clos de douze matrices, dont dix reprises de Tournier (préfixation, suffixation, dérivation inverse, composition, amalgame, onomatopée, conversion, troncation, siglaison et emprunt) et deux ajoutées: la déformation, pour rendre compte des argots à clef français comme anglais (verlan, largonji, javanais, backslang, Pig Latin, voir Antoine [1999]) et le jeu de mots (purement formel, par jeu sur la proximité phonétique ou graphique, mais l'usage a montré que le sémantique intervenait souvent aussi dans les jeux de mots). Idéalement, cette machine à créer du lexique comporte un troisième axe, temporel, certaines combinaisons ou certaines matrices étant particulièrement actives ou productives à des périodes données - mais la datation des argots est notoirement difficile à établir, puisqu'elle se fonde historiquement sur l'enregistrement de leurs créations à l'écrit, qui peut être précédé d'une longue existence à l'oral uniquement (ceci est de moins en moins vrai à partir du quatrième quart de XXe siècle, où argotologues et lexicographes ont commencé à enregistrer les nouvelles créations de façon de plus en plus efficace et quasi instantanée). En d'autres termes, ce modèle de machine dissocie le morphologique et le sémantique, pour montrer qu'ils se combinent, dans au moins la moitié des items d'un champ donné (Antoine [2004]) là où Tournier et Sablayrolles n'envisagent qu'une catégorie morpho-sémantique parmi d'autres.

7 La machine montre que certains items relèvent purement du formel: un mot qui désigne un objet est par exemple tronqué ou suffixé, ou tronqué puis suffixé - son sens ne change pas. Par exemple, le mot « flic » (qui, par son sémantisme d'origine, au début du XIXe siècle, est lié à « mouche » (pour mouchard, espion) via l'allemand Fliege puis le yiddish) est transformé, déformé, en javanais près d'un siècle plus tard en flavic (1912) et ce n'est qu'un des avatars de ce mot. D'autres items relèvent purement du sémantique (un mot est remotivé mais sa forme ne change pas). Par exemple, le mot " perdreau » prend le sens de " policier » (1952), sans changer de forme, mais en étant remotivé pour le rapprocher, sémantiquement, du déjà ancien et usé " poulet » (1933) (qui a lui-même subi plusieurs déformations), lié sémantiquement à "poule " pour « police » (1899), vraisemblablement emprunté à l'italien pula (et déformé) (même sens) - « perdreau » est ensuite déformé en « drauper » (1953) : c'est du formel.

8 Les exemples qui précèdent montrent d'une part que, dans les argots, des matrices formelles peuvent être mises en jeu successivement, au fil du temps, sans affecter le sémantisme de l'équation métaphorique ou métonymique qui forme la matrice sémantique, par exemple "policier = volatile", sans doute dérivée de "policier = idiot ", ou "policier = mouchard/espion». D'autre part, que différentes matrices sémantiques peuvent être sollicitées selon les périodes, selon la transparence de celle utilisée auparavant, selon le sème qui est haussé à tel ou tel moment de l'histoire, au gré de la vision du créateur, au gré de l'image mentale qu'il superpose à celle de l'objet de départ, ici, le policier, en combinaison ou non avec une matrice formelle. Selon que tel créateur (ou telle époque) voit plutôt le policier comme un individu aux méthodes brutales ou comme son enveloppe (son uniforme, ou un élément de celui-ci), alors certains mots sont remotivés (heavy, headbeater, « cogne ", "bigorne " / copper, helmet, " képi ", "pèlerine »), non sans humour, même caustique, même amer, puis ils peuvent être remplacés par d'autres au fil de leur perte d'opacité (voir les dates d'apparition) ou simplement parce que le jeu de la création lexicale argotique fait qu'il y a une sorte de surenchère. Tel locuteur choisit-il de privilégier le couvre-chef du bobby (d'où 
l'équation «bobby = son casque ») ? Le saut métonymique permet de remplacer bobby par une expression qui rime avec une autre désignation, elle aussi métonymique (le matériau des boutons caractéristiques de son uniforme), qui est copper et l'on obtient le rhyming slang bottle and stopper (1928): l'image mentale du bouchon de liège (ce matériau entrant dans la composition dudit casque) qui surmonte une bouteille plus ou moins ventrue est superposée à celle du policier en uniforme, du bobby un tant soit peu bedonnant « surmonté » de son casque. La superposition prête à sourire, tout comme la création lexicale qui, dans son avatar suivant, est tronquée en bottle. Plus tard, comme une sorte d'écho, on a bottletop et spinning top (1944), puis cozzpot (1962), puis woodentop (1981), puis woody (sd): on voit la filiation et comme une sorte de ricochet d'une désignation à l'autre, ou de rebond, comme si les créateurs s'appuyaient sur un mot pour le déformer un peu et en obtenir un autre qui porte leur marque et éventuellement se remotive partiellement au passage (croisement avec d'autres équations, comme "policier = idiot", par exemple). Il en va de même avec la série scufter / walloper / paddler / muzzler / headbeater, où des verbes signifiant tous " frapper » sont suffixés en "-er» (= one that $V$ ) et sont mobilisés pour désigner le policier "cogneur». En français, on a la série "cogne » (1800), "sonne » (1881), «bourre» (1910) (qui donne «bourrman» et «bourrich», [sd]), «bourrin» (1954), " cognac » et «cognard» (sd), «chtar» (sd), " pot à tabac» (sd) - même s'il est probable que « bourre » ne porte pas d'abord le sème |frapper| mais est la troncation de «bourrique » (1877), qui désigne par antiphrase la monture du gendarme à cheval et donc par métonymie le militaire lui-même (mais le sème |têtu| est sans doute là); l'attraction de «bourrer de coups » renforce l'équation métaphorique démontrée par les autres mots : on voit que le rebond peut se combiner à d'autres matrices.

\section{Productions en séries}

Les exemples ci-dessus, malgré leurs ramifications, montrent que la création lexicale par rebond est très productive en argot, par nécessité d'abord sans doute (de passer à un mot plus opaque que le précédent, devenu trop transparent), par pur jeu ensuite probablement, pour démontrer une virtuosité à créer et pour ranimer en quelque sorte la virtualité humoristique de la désignation, née de l'image mentale convoquée par le jeu sur les mots, par l'équation métaphorique actualisée. Ce phénomène de rebond donne naissance à des séries observables dans les argots de l'anglais comme du français, selon l'une ou l'autre des trois méthodes suivantes.

\subsection{Rebond à partir d'une matrice sémantique}

Ici, la dénomination de tout objet ou de toute notion qui comporte le sème haussé dans une équation métaphorique peut-être mobilisée pour désigner le premier terme de cette équation : si «argent = couleur jaune » (à cause de l'or, à l'origine), alors tout objet plus ou moins jaune peut désigner de l'argent («blé », etc.), en particulier ce qui est doré ("galette», " pognon», " douille»), plus encore si une autre équation vient renforcer cela ( argent = pain» > «blé »; «argent = objet rond et plat» 》 "galette »). C'est de cette façon que la notion de "travailler " peut s'exprimer en argot et en langue familière grâce à une quantité de verbes, tels que, par exemple, «boulonner, bûcher, buriner, gâcher, piocher, trimer, turbiner, usiner » ou, en anglais, 
dig, graft, grind, parce que "travailler = se livrer à son métier " et donc "effectuer l'action principale ou typique dudit métier", et l'on reconnaît des outils ou des produits de diverses professions dans les séries ainsi générées ${ }^{3}$ - une autre équation est «travailler = se fatiguer, s'épuiser ", qui génère à son tour une série de verbes portant ce sème pour dire la même chose (si la première matrice est moins productive en anglais, la deuxième l'est en revanche davantage qu'en français). Ainsi chaque métier voit-il en quelque sorte midi à sa porte et parle de l'action de travailler en fonction de ce qu'elle implique pour lui : rien de plus naturel que de ramener le monde à soi. De la même façon, et selon le même mécanisme, chaque métier va "inventer ", créer, des désignations d'objets ou d'actions en fonction de son univers familier; un exemple typique est la série de désignations de l'acte sexuel fondées sur la mise en avant du sème |mouvement de va et vient|, en fait, la réduction à ce sème, que l'on pourra juger périphérique plutôt que central, c'est-à-dire non susceptible de faire partie de la définition de « coït » ou " copulation », par exemple. Chaque métier va ainsi remotiver un verbe d'action qui lui est propre, qui implique, est ou évoque un mouvement de va et vient, ce qui donne la série " artiller, brosser, caramboler, égoïner, limer, ramoner, etc. » ou, en anglais, dig, grind, poke, pound, pump, shag, saw a chunk off, etc. - étant bien entendu que là n'est pas la seule équation métaphorique ou métonymique mise en œuvre, évidemment, loin de là ; l'auteur de la remotivation d'un verbe "de métier » livre un commentaire sur son activité en même temps qu'une vision décalée de l'acte sexuel.

\subsection{Rebond à partir du sémème d'un objet}

11 Ce deuxième type de rebond s'opère en haussant un sème différent pour activer chaque fois une équation métaphorique ou métonymique nouvelle, combinée ou non avec une matrice formelle. Par exemple, un couteau est constitué d'une lame, pointue, plus ou moins longue et se manie à la main, au bout du bras tendu, éventuellement, dans un combat - les sèmes repérables dans cette définition partielle de la forme de l'objet permettent de créer des désignations de celui-ci en argot : «lame» (1889), " pointe » (1957), « scion » (1878), « vingt-deux » (1828), « rallonge » (1894). Des sèmes en rapport avec la fonction de l'objet permettent d'autres créations : « lardoire » $(1842$; le couteau peut servir en cuisine à larder une viande), « cure-dents » (1921), « silencieux » (1918; il peut tuer sans bruit), «vendetta » (1962). On peut considérer qu'il évoque l'épée des chevaliers ou des mousquetaires, et c'est un "Charlemagne » (1872) ou une " rapière " (1928); on peut emprunter sa désignation au tzigane ("surin», 1827), à l'italien (" sacagne », 1899), à l'anglais (" schlass », 1932) ou l'on peut conserver un nom propre en l'appelant "Eustache " (1818; de Eustache Dubois, coutelier de son état, à SaintÉtienne, au XVIIIe siècle). On aura remarqué que les matrices sémantiques peuvent, selon les cas, se combiner avec des matrices formelles (conversion, emprunt). L'anglais s'appuie sur la forme de la lame, pointue, effilée (blade, 1896, edge, 1972, shank, 1974, thorn, sd), compare avec d'autres objets tranchants (ax, 1896), met en vedette le cran d'arrêt (switch, 1941), des fonctions auxquelles ne pense pas forcément toujours le français (frog-sticker, 1836, toad-sticker, 1863, pig-sticker, 1870, California toothpick, 1856, Harlem toothpick, 1944, slasher, 1815 (mais 1673 dans le sens de « épée »), carver, 1907, gut-ripper, 1947, borer, 1997, onion-peeler, 2000, tool, 1834, où l'on reconnaît un jeu cruel d'enfants, la boucherie ou la cuisine entre autres) ; par ailleurs, il emprunte au Romani (chiv, 1674, qui signifie « lame ») ou passe le mot au filtre d'argots à clef : April fool, (sd), 
charming wife (1925), drum and fife (1925) qui donne drummond en rhyming slang (riment avec tool et knife, respectivement), ou efink (1859) en backslang. Et ce n'est qu'une petite sélection de créations lexicales parmi plus de 160 désignations relevées dans le dictionnaire de Jonathon Green. On constate que l'anglais et le français sont comparables même si non superposables, et l'on admettra que l'humour de ces désignations naît de la superposition d'images mentales plus ou moins inattendues: c'est le décalage qui génère l'humour, certes quelquefois relatif ; c'est le jeu entre ce que le mot ou l'expression fait visualiser spontanément et le nouvel objet qu'on lui fait désigner, très partiellement, très partialement, le couteau. Le créateur, en nous entraînant à voir l'objet à sa façon très particulière à lui, nous incite à " rire avec » lui, invite notre esprit à reconnaître ce jeu et à l'apprécier intellectuellement. On souligne que les sèmes sollicités peuvent être centraux (font partie du sémème nécessaire et suffisant pour une définition de l'objet) ou périphériques (au-delà de la définition, utiles à une définition hyper-spécifique, encyclopédique dans sa nature $)^{4}$; plus les sèmes sont périphériques, plus la désignation est inhabituelle, inattendue et susceptible d'être perçue comme humoristique: il y a plus d'esprit à nommer un couteau un "silencieux » ou an onion peeler qu'à le nommer, très prosaïquement, une «lame» ou a blade.

\subsection{Rebond à partir d'une matrice formelle}

Le rebond se fait, dans une troisième méthode, à partir d'une des matrices formelles voir les déformations successives de "flic» (une quinzaine) ou de "poule » (13), de detective en anglais (8) - ; on tronque un mot déjà tronqué, on le suffixe différemment, on le reverlanise après l'avoir tronqué, etc.: c'est là une forme de surenchère permanente, à distance, entre créateurs et utilisateurs des argots. C'est par exemple ce que l'on relève dans des séries comme «trad/trado/tradal/traduc (?)» (pour dire «traduction », dans l'argot universitaire) ou « pistoche/piscaille/pistache » (pour dire " piscine ", dans l'argot des écoles), où l'on reconnaît les procédés purement formels de la troncation, la suffixation (à l'aide de suffixes classiques, productifs, comme «o ", hypocoristique et/ou diminutif, ou de ce que Pierre Guiraud [1980:72] appelle « queues postiches ", du loucherbem (-oche) ou non (-aille), le jeu de mots (purement formel: par écho et rebond sonore, " pistAche ", «tradAL » (si l'on applique à rebours la règle selon laquelle les mots en -al font leur pluriel en -aux ([o]) !).

Les deux premières variétés sont parfaitement classiques, y compris pour le lexique général, même si elles y sont moins développées qu'en argot - il s'agit de remotivations en cascade. La troisième variété est, me semble-t-il, davantage propre aux argots et à la langue familière et elle reflète particulièrement leur créativité. Je souhaite l'illustrer à partir de corpus (au sens de "sac de mots et d'expressions $»^{5}$ ) réunis et exploités par trois étudiantes dont j'ai dirigé les récents travaux de mémoire de master, que je sollicite donc ici, car ils sont particulièrement aboutis et finement analysés. 


\section{Jeux de formes : études de cas}

\subsection{Jeux de formes : mots isolés et composés}

14 Dans une étude des désignations de la lesbienne en argot et en langue familière qui s'appuie sur un ensemble de 389 mots et expressions de l'anglais et un ensemble de contrôle et comparaison de 106 mots et expressions du français (Bécart [2020]), ont été repérés les nombreux sèmes haussés pour créer ces désignations (l'ensemble des sèmes, hiérarchisables, permettant d'écrire une définition très développée, hyper-spécifique, du mot «lesbienne » et de "raconter», de «narrer ", la façon dont on a parlé des lesbiennes à travers les époques, ce que ce mémoire fait admirablement). Parmi ceuxlà, évidemment le sème |attracted to women| et le sème |that performs cunnilingus| (en réalité un sous-sème de |has sexual intercourse with women|).

Si l'on considère le premier sème, on constate une sorte d'emballement de la machine à créer du lexique, dans lequel le mot lesbian est déformé de toutes sortes de façons, avec le même but, créer une forme nouvelle, originale (ou voulue comme telle) et propre à attirer l'attention, voire à faire admirer la virtuosité du créateur, sa hardiesse même, quelquefois son humour ou son esprit, car certains items sont des clins d'œil. On dénombre ainsi, entre 1925 et 2003, trente avatars de lesbian, de lezo (1925) (mais lesbo (1927), les (1929) et liz, (1928)) à lezza (2002) ou lebanese (2003) ou les-be friends (sd). On admettra que la machine tourne un peu à vide ici, de façon mécanique (le sémantisme de la série est même sans doute neutralisé, le lien étymologique avec Lesbos étant perdu: on n'a ici qu'un jeu répétitif sur la forme). L'humour est ténu, mais la série montre le fonctionnement d'une machine à créer le lexique uniquement sur le plan formel, en mettant en jeu les matrices de la troncation, la suffixation et le jeu de mots (purement formel).

Le deuxième sème, |that performs cunnilingus|, entre 1890 et 2003, est le sème unique de trente items différents (7,72\% du corpus), tous, à l'exception de deux, des composés dont la forme est X-Y-er, où $\mathrm{Y}$ est un verbe synonyme de eat (la grande majorité) ou lick ou suck et, marginalement, tap, et $\mathrm{X}$, une désignation argotique du sexe féminin (cunt, fanny, muff, crack, etc.) ou de la toison pubienne (carpet, rug, etc.), le suffixe -er servant à nominaliser le verbe pour désigner l'auteur de l'action. Ceci répond exactement aux 22 items du corpus français, sur le modèle correspondant de "Y-euse de X ", où Y est le même type de verbe qu'en anglais. On voit, en examinant les dates d'apparition, que les items se construisent par écho, par surenchère, par rapport aux précédents, des premiers répertoriés, licker (1890), sucker (1899), muff diver, et cunt lapper respectivement de 1930 et 1934 aux derniers cunt muncher, (2003) ou carpet biter (2002). La machine à créer du lexique semble ici tourner automatiquement, encore une fois, à partir d'une forme donnée, dans laquelle l'un ou l'autre élément ou les deux peuvent être permutés pour produire une nouvelle dénomination mais sans que le sème ne soit gommé ou estompé : la forme est en quelque sorte répliquée comme pour en fournir une nouvelle expression, toujours nouvelle, mais toujours voisine. On admettra que la hardiesse des images mentales évoquées n'en est pas la qualité principale, mais je suis convaincu que le créateur d'un nouvel item voit celui-ci comme une marque d'esprit, personnelle et empreinte d'un certain humour. Ce deuxième exemple, jouant sur des composés, offre plus de possibilités d'évocation d'images décalées que le premier, où le jeu sur la forme se limite à un mot isolé. 
17 On retrouve le même type de fonctionnement dans une étude des désignations du gros (Silberstein [2020]), où, à partir d'un corpus de 300 mots et expressions en anglais et d'une centaine en français, l'on peut repérer des séries plus ou moins développées, par exemple de compositions ou de suffixations sur blubber ou lard, dans lesquelles c'est un humour cruel et grossier qui s'exprime aux dépens de la personne grosse. On peut ainsi suivre la façon dont lard est repris et dont la surenchère s'exerce dans la série lardy (1879), lard bladder (1893), lard arse (1918), lard can (1924), lard belly (1930), lard ass (1937), lard ball (1949), lard bucket (1958), lard sack (1968), lard butt (1971), lardo (1987), lard batty (2000 env.). Ici, la matrice sémantique alterne entre partie du corps (métonymie) et contenant (métaphore). On retrouve la deuxième matrice sémantique dans une autre série, qui est une composition du type tub of $\mathrm{X}$, où $\mathrm{X}$ désigne de la matière grasse (lard, 1835, ou blubber, 1994), les entrailles (guts, 1837) ou de l'excrément (turds, 1660, shit, 1974). On conviendra qu'il s'agit ici, dans cette série de surenchères, d'autant de saillies faites pour " rire contre » la cible, la blesser, et certainement faire rire ou sourire à peu de frais le spectateur.

\subsection{Jeux de formes : lignes parallèles}

18 Un autre corpus permet de voir une série très riche, sans l'humour cruel des précédentes, mais avec une touche d'humour bienveillant, qui sert à aborder, comme souvent en argot, un sujet tabou de façon détournée, voilée. La convocation d'images mentales décalées contribue à cela.

Il s'agit ici d'une étude des mots et expressions utilisés en anglais, en argot et en langue familière, pour parler de la menstruation et des règles, appuyée sur un corpus de 556 items en anglais et 213 en français (Dufour [2020]). Huit sèmes centraux ont été dégagés, tels que |blood|, |protection| ou |periodicity|, chacun étant issu de la définition standard des dictionnaires, légèrement élargie (ce qui justifie |protection|, par exemple) et chacun étant subdivisé en plusieurs sous-sèmes ; par exemple, |blood| se distribue en | blood colour|, |blood staining|, |blood texture|, |blood flow|, |blood injury|, ou |periodicity| en | regularity|, |duration|, |unexpectedness|, |lateness| ou |visit| (qui est métaphorique, mais l'on comprendra que les sèmes visent à nommer une notion, une idée associée et non une chose). Les expressions qui contiennent month ou dérivés, period, moon, time (éventuellement combinés : that time of the month, réduit par siglaison en T.O.M.), par exemple, se rangent sous |regularity|.

On voudrait s'intéresser au sème |visit|, qui réunit un bon nombre d'euphémismes pour parler de ce sujet dont on rappelle qu'il est tabou. On trouve 46 items où ce sème se réalise de façon presque banale (have a friend visiting / a visitor / company / a country cousin, the visitations, Kit has come, etc.), où le sourire qui accueille la périphrase ou l'accompagne est davantage de connivence que pour souligner ce qu'elle peut avoir de décalé ou d'insolite; cependant, on relève tout de même une sorte de surenchère ici, où le(s) visiteur(s) reçoi(ven)t une nouvelle identité, sans doute voulue plus originale, à chaque itération. Mais ce sème entre en combinaison avec d'autres, pour fournir une quantité d'expressions où se décèle davantage d'humour, par l'originalité ou la complexité de la formule créée, par la volonté d'inventer une variante inattendue ou qui renouvelle une expression connue, jeu, ici, bien sûr, qui donne une épaisseur humoristique à ces expressions. La première combinaison se fait avec des sous-sèmes du sème $|b l o o d|$, dont le prototype semble être the cardinal is come, où the cardinal se 
rattache au sous-sème |blood colour| et is come à |visit|, et semble être apparue au milieu du XVIIIe siècle. Les mêmes sèmes ont été, en quelque sorte, réactivés au milieu du XXe siècle, avec des expressions comme the Campbells are coming (1948), Grandma is here from Red, Aunt Emma from Reading (1948), my red-head aunt from Redbanks (1948) ${ }^{6}$, et l'expression source elle-même est recyclée en 1980 avec the Cardinal is home et en 2000 sous la forme a visit from the cardinal. Mais c'est le dernier quart du XXe siècle et le début du XXIe qui voient une explosion d'expressions qui surenchérissent les unes sur les autres en utilisant les deux sèmes en question, avec, par exemple, a visit from the rose fairy (2005), a red-headed friend (1999), the Red Sox are in town (2001), red-head aunt from the South (2000), etc. On voit que d'autres sèmes sont actualisés dans certaines de ces expressions, la référence au bas du corps (South) ou celle à une figure maternelle, comme la tante (on a aussi la grand-mère), sans doute parce que c'est une femme à qui se confier et à même de donner des explications rassurantes. Et l'on relève quantité d'expressions qui rivalisent de créativité et prêtent à sourire, bâties sur le mot aunt. En voici un échantillon: tout d'abord, Aunt, suivi d'une variété de prénoms féminins, dépourvus de double sens, non connotés, comme Irma (2009), Jane (1948), Susie (1948), Tillie (1999) ou avec jeu de mots (et références à autres sous-sèmes : |colour|, |staining|, | flow|) : Rose (1999), Ruby (2002), Flo (1974), puis à partir de là, on construit, on développe, on surenchérit: Aunt Flo from Red China (2003), Aunt Flo and Cousin Red (2009), Aunt Flo from Red River (2007) / from Redland (2000), my aunt from Reading (2000) / from Redfield (2000) / from Redwood City (2000), etc. Et puis il y a les variantes, qui divergent par un élément ou un autre : Flo is coming to town (2007), Cousin Dotty (2010), Carrie (2018), Uncle Bloody (2001), les variantes sur les communistes, etc. On constate que l'imagination se donne libre cours, la fantaisie aussi, et chaque créateur/créatrice ${ }^{7}$ apporte sa contribution au stock lexical, avec, ici, si l'on compare au corpus précédent, une touche humoristique plus souriante, plus " gratuite », dans le sens où l'humour ne s'exerce pas aux dépens d'un(e) autre : il s'agit bien ici de « rire avec ». Et l'on souligne que, une nouvelle fois, la machine semble tourner sur une forme que l'on module à volonté, en en permutant au moins l'un des éléments, mais en écho, et en combinaison avec les sèmes activés, par les équations métaphoriques ou métonymiques du champ qui font que tel sème est mis en avant, représente à lui seul l'objet considéré.

On constate que la machine, du côté du français, est moins productive et met en jeu moins de sèmes différents. Le sème |visite| n'est repérable que dans une quinzaine d'expressions, de "voir Sophie » (1867; y a-t-il un lien avec Les malheurs de Sophie de 1858 ?) à «avoir de la visite " (1981) en passant par «avoir Martin» (1901) ou «avoir les parents à Montrouge » (1948), qui est la seule expression du corpus à associer |visite| à |couleur du sang| et à marier en même temps l'euphémisme à un jeu de mots. On observe que la figure maternelle n'apparaît pas en français, ni la figure féminine éventuellement amicale, ce qui fait que les jeux sur des prénoms sont absents. C'est la référence au sang qui domine le corpus avec 90 items sur 213 (42,25\%), dont plus de la moitié par référence à la couleur rouge (56 items, 26,29\%). Conformément à ce que l'on observe régulièrement dans les argots, ici, tout ce qui possède le trait retenu, |couleur rouge|, est potentiellement mobilisable pour parler de l'objet considéré, les règles; la machine, en français, ne recycle pas une forme en combinaison avec un ou plusieurs sèmes : elle produit de nouvelles expressions en jouant de la même matrice sémantique, selon le premier type de rebond décrit plus haut (2.1.), « règles = sang » et même, plus lapidairement, "règles = couleur rouge ». Le prototype semble bien être la toujours connue « les Anglais ont débarqué » ou « avoir ses/les Anglais » (1850, en raison de la 
couleur rouge de l'uniforme des soldats anglais, les 'redcoats' pour l'anglais), mais la pourpre cardinalice inspire également, comme en anglais, avec « les cardinales » et « le cardinal est logé à la motte » (1850 aussi, la deuxième expression se doublant d'un jeu de mots). Le croisement avec les sèmes |liquide| ou |tache| permet des expressions qui font référence à des fruits ou légumes rouges (tomates, groseilles, fraises : « écraser des tomates" est enregistré en 1850) ou des fleurs rouges (coquelicot, rose rouge). On observe d'ailleurs que "fleurs " a été une désignation des règles, datant au moins de 1360 (DMF), d'abord dans le vocabulaire médical puis en langue courante, avec valeur euphémique. C'est ainsi que le mot apparaît dans les dictionnaires de Richelet [1680], Furetière [1690], de l'Académie [1694] : «Fleurs au pluriel sign. aussi, Les ordinaires des femmes. ", mais aussi dans le dictionnaire bilingue de Cotgrave [1611]: «a womans monethlie flux, or flowers »), tout comme flowers (même sens, daté par OED d'environ 1400 , emprunté au français). Le mot français est la déformation de "flueurs " (1362, $D M F)$, au sens de "écoulement ", directement emprunté au latin fluor et même menstrui fluores; la proximité phonétique a évidemment contribué à la déformation de "flueurs " en "fleurs " mais il semble aussi indubitable que le sème |tache|, et peutêtre aussi |rouge|, a autorisé cet emploi métaphorique de « fleurs ", en une combinaison de sémantique et de formel somme toute classique. La métaphore a ensuite été empruntée par l'anglais et recyclée dans les deux langues (decorated with red roses, 1999). Tout objet rouge, enfin, peut être saisi par une expression, le rubis, le rouget, le ketchup, le Beaujolais nouveau, le drapeau rouge (du chef de gare ou de la Commune), le mot ou le panneau STOP, le feu rouge, les chemises rouges, etc., jusqu'à " repeindre sa grille en rouge " (1981) ou " au minium » (2009). On le voit, on a en français une variation sur |rouge|, en combinaison ou non avec d'autres sèmes, et la surenchère sur une forme que l'on peut régénérer à l'envi n'existe pas comme en anglais, ce qui laisse moins de champ à la créativité et certainement à l'insolite, aux jeux de mots en surenchère, comme observé plus haut (2.1.).

On pourrait sans doute trouver certaines expressions produites moins convaincantes, moins réussies, moins décalées que d'autres, et c'est certainement vrai : la machine à créer du lexique ne produit pas des items d'égale qualité - la surenchère peut tourner court. Mais elle peut aussi, selon les champs, toujours à partir d'une même forme, produire de longues séries d'expressions également insolites, inattendues, empreintes d'humour.

\subsection{Jeux de formes : l'itération sans fin?}

C'est le cas d'un ensemble d'expressions qui désignent la bêtise d'un individu. Le corpus réuni (Sauvage [2014]) comprend 280 expressions (et s'accompagne d'un corpus de 303 mots). Être bête, cela peut être, principalement, être |vide| (avoir le cerveau vide), être | incomplet| (il manque quelque chose à l'individu, quelque chose d'essentiel) ou être | défectueux| (avoir un défaut de fonctionnement). Les trois équations autour de ces sèmes rendent compte des trois quarts des expressions réunies, avec la part du lion pour la deuxième, |incomplet| (37\% du corpus). Trois formes d'expressions apparaissent pour exprimer ce sème, dont deux sont des antiphrases, à la portée humoristique déjà naturellement présente ${ }^{8}: 1$ ) NOT THE X-EST Y IN THE $Z$, où X est un adjectif qui, au sens figuré, peut vouloir dire « intelligent» (bright, sharp), Y est un nom auquel l'adjectif au sens propre s'applique et $Z$ un nom du paradigme de $Y$; le type en est not the sharpest knife in the drawer ; 2) DOESN'T HAVE, suivi de ALL X IN/ON Y, où Y est le lieu où se trouve en 
général un ensemble complet de $\mathrm{X}$; le type en est doesn't have all the dots on his dice, mais il existe quelques variantes du type doesn't have a round in every chamber. Ces deux séries, de 6 et 14 expressions respectivement, peuvent donc être adaptées, au gré de l'utilisateur qui veut leur donner une nouvelle vie, une originalité qu'elles n'ont plus à ses yeux, un côté spirituel personnel; elles sont à disposition, formes prêtes à être redynamisées, voire subverties, en écho. On a en français une production comparable d'expressions à partir de la forme "il n'a pas inventé... », où le complément peut être « le fil à couper le beurre, la roue, l'eau tiède, l'eau chaude, la poudre, l'eau en poudre, la machine à cambrer/cintrer/courber/couder les bananes ", etc. (Bernet \& Rézeau [2008]). On dira que l'humour du français est plus farfelu, frise l'absurde, finalement le nonsense à l'anglaise, mais la réduction d'un individu à un objet, en anglais, va aussi dans ce sens.

C'est dans la troisième forme que l'insolite des images évoquées est le plus fortement présent, en conjuguant l'ancrage dans la réalité (rurale, technique, quotidienne, etc., expérientielle, pour évoquer Lakoff \& Johnson [1985] et leur lecture de la métaphore ${ }^{9}$ ) et une virtuosité dans le renouvellement de la forme, qui est A FEW X SHORT OF Y, où X est un élément de l'ensemble désigné par $Y$, sans lequel Y n'est pas complet, clos. Cette forme se retrouve dans pas moins de 77 expressions du corpus (soit $28 \%$ du total) et le prototype en est vraisemblablement une forme moins élaborée, short of a sheet of bark (1890), qui évoque une toiture incomplète (donc non étanche). On trouve plus tard (1919) three pence short of a shilling et a few pence short in the shilling (1932), qui font écho à only ninety-nine cents out of the mental dollar. Les expressions liées à l'argent ont été remodelées pour parvenir à la forme qui a permis de générer toutes les expressions évoquées, en allant vers des ensembles de moins en moins formalisés et stables et donc des expressions de plus en plus loufoques. En voici quelques-unes : $a$ few inches short of $a$ foot, a few peas short of a casserole, a few cards short of a full deck, a few feathers short of a duck (!), a few clowns short of a circus, et les variantes suivantes : a bun short of a dozen, a couple sandwiches short of a picnic, two sheep short of a sweater, a sausage short of a barbecue, one shingle shy of a roof - mais l'on constate que ces variantes respectent le profil général de la forme de base ${ }^{10}$. On le voit, si certaines expressions sont analysables selon les règles du bon sens et de la réalité connue, d'autres nécessitent en quelque sorte de combler les trous de la chaîne logique qui les sous-tend: ce sont celles-là qui sont les plus inattendues, les plus savoureuses, dont l'humour est le plus susceptible de faire réagir celui qui les entend, qui est invité à « rire avec » son créateur.

Un peu comme dans le cas précédent, où les itérations autour de prénoms ou de noms de lieux sont à même d'évoquer, au moins phonétiquement, la couleur rouge ou l'écoulement, ici, la machine fonctionne à partir d'une forme reprise et modifiée à l'envi. Dans le cas précédent, il s'agissait de créer un nouveau jeu de mots sur le modèle des précédents; on peut donc penser qu'il y a une certaine épaisseur dans cette création lexicale, qui cherche aussi à rebondir, à surenchérir, sur l'humour de la production précédente. Ici, avec la même visée humoristique, sans véritable recherche de jeu de mots, la machine reproduit la même forme strictement (une fois qu'elle a été stabilisée) : il suffit au créateur de trouver deux termes $\mathrm{X}$ et $\mathrm{Y}$, dont le premier est un élément de l'ensemble du deuxième, pour générer une nouvelle expression, plus ou moins cohérente, plus ou moins hardie, ou astucieuse, ou même forcée pour certaines mais ce côté « forcé » fait le loufoque et donc le décalage, l'inattendu de l'expression et donc en renforce l'humour. Ce fonctionnement me semble plus mécanique car moins 
contraint, ce qui se reflète aussi dans sa plus grande productivité (plus de quatre-vingts formes, 'and counting', comme l'on dirait en anglais: tous les ensembles d'objets peuvent être légitimement mobilisés dans cette expression, selon l'envie du créateur, mais aussi selon ceux qu'il envisage spontanément, en fonction de ses intérêts et de sa réalité ${ }^{11}$. Ce qui fait que l'expression est comprise, qu'elle résonne pour celui/celle qui l'entend, ce n'est pas tant l'écho, plus ou moins comique, entre $\mathrm{X}$ et $\mathrm{Y}$, mais précisément la forme A FEW... SHORT OF, qui dit dumb/bête, à cause des itérations dont elle a fait l'objet, toujours sous la même équation métaphorique : la forme implicite le sens, au point, d'ailleurs, que le lien posé à l'origine entre $\mathrm{X}$ et $\mathrm{Y}$ peut se distendre, ce qui contribue au loufoque de certaines expressions - le cirque est-il en effet, un ensemble de clowns ou doit-il comprendre un nombre donné de clowns pour exister ? Le cookie doit-il de la même façon contenir un nombre précis de pépites de chocolat? Un canard a-t-il un nombre fini de plumes, sans lequel il n'est pas un canard? Ainsi de suite : la forme, indépendamment des éléments qu'elle met en relation, dit ce que son prototype disait.

\section{Conclusion}

«Un argot est l'ensemble oral des mots non techniques qui plaisent à un groupe social " ${ }^{12}$, écrivait Gaston Esnault en 1965 dans la préface de son Dictionnaire historique des argots français. Le mot ou l'expression argotique a une carrière s'il ou si elle plait au groupe auquel appartient son créateur, puis à d'autres groupes; il ou elle est repris/e et diffusé/e s'il ou elle trouve un écho chez ceux qui l'entendent. En cela, il pourrait être défini comme Le Guern [1973:82] le fait de la métaphore: "L'évolution historique d'une métaphore peut se schématiser ainsi: création individuelle, dans un fait de langue d'abord unique puis répété, elle est reprise par mimétisme dans un milieu précis et son emploi tend à se généraliser dans la langue "; les argots sont métaphore, se créent et s'organisent selon des équations métaphoriques ou métonymiques qui les structurent en se combinant ou non avec des matrices formelles. Chaque groupe social et même chaque individu à l'intérieur de celui-ci, est fondé à créer sa propre désignation pour tout objet ou toute notion qu'il veut distinguer, c'est-à-dire qui appartient à son environnement immédiat, lui est utile, précieux, intéressant, etc. Ceci explique le foisonnement et le renouvellement des argots, qui se font selon un ensemble de règles évoquées plus haut et, de plus en plus, avec une dimension ludique affirmée, où c'est le décalage, même infime, qui fait que l'autre reconnaît et goûte intellectuellement le nouveau mot ou la nouvelle expression.

Les séries présentées dans les pages qui précèdent illustrent la manière dont la machine à créer du lexique argotique fonctionne et montrent que le fonctionnement le moins "onéreux", qui demande le moins d'effort, est aussi le plus mécanique, qui repose sur les matrices formelles (en nombre limité) uniquement, et même sur une matrice formelle unique, réitérée encore et encore. Lorsqu'il s'agit d'expressions, c'est alors une forme qui subit des glissements successifs, à partir d'un prototype qui peut finir par être perdu de vue. Les argots fabriquent ainsi du nouveau, de l'original, de l'inattendu, à partir de matériau existant, réputé connu de tous, et le ressort qui meut cette machine, comme j'espère l'avoir montré, est l'humour : on est ici en plein dans la fonction ludique des argots et dans une sorte de surenchère constante où chaque 
création est une tentative d'avoir le dernier mot, le plus amusant, le plus frappant, le plus humoristique.

\section{BIBLIOGRAPHY}

ANTOINE Fabrice, 1999, « Verlan français, backslang anglais, etc. », Cahiers de lexicologie (CNRS) $\mathrm{n}^{\circ} 74-1,171-183$.

ANTOINE Fabrice, 2001, « Lexique de l'argent et passage à l'euro », French Studies Bulletin n ${ }^{\circ} 79$, 17-19.

ANTOINE Fabrice, 2004, « Argots et langue familière : quelle représentation en lexicographie bilingue ? » in ANTOINE Fabrice (Ed.), Argots, langue familière et accents en traduction, Lille : Cahiers de la Maison de la Recherche, Ateliers n³1, 11-23.

ANTOINE Fabrice, 2008, « De la thune à la caillasse : argent, argots et langue familière » in VATANPOUR Sina (Ed.), L'argent, Villeneuve d'Ascq : Collection UL3, Université Lille 3, 329-339.

ANTOINE Fabrice 2011, « Argots : histoires de métaphores et de métamorphoses », Les Cahiers du Dictionnaire $\mathrm{n}^{\circ} 3,63-82$.

ANTOINE Fabrice, 2014, « Les langues de spécialité sont-elles des argots comme les autres? Convergences/divergences, enjeux lexicaux, stylistiques et traductologiques ", ASp n66, 7-25. BÉCART Camille, 2020, A Comparative Study of English and French Words and Expressions Used to Designate the Lesbian in Slang and Colloquial Language, Mémoire de master de Traduction et interprétation, Parcours MéLexTra, Université de Lille, sous la direction de F. Antoine. BERNET Charles \& RÉZEAU Pierre, 2008, On va le dire comme ça - Dictionnaire des expressions quotidiennes, Paris : Bardolle, Gawsewitch et Naulleau Éditeurs, Balland.

CALVET Louis-Jean, 1994, L'argot, Paris : PUF, 'Que Sais-Je’.

COTGRAVE Randle, 1611, A Dictionarie of the French and English Tongues, Londres: Adam Islip.

DUFOUR Chloé, 2020, A Study of Menstruation in English Slang and Colloquial Language, Mémoire de master de Traduction et interprétation, Parcours MéLexTra, Université de Lille, sous la direction de F. Antoine.

DMF : Dictionnaire du Moyen Français (1330-1500), version 2015 (DMF 2015). ATILF - CNRS \& Université de Lorraine., consulté le 12 mars 2021, http://www.atilf.fr/dmf ESCARPIT Robert, 1991 (1960), L'humour, Paris : PUF, ‘Que Sais-Je ?’.

ESNAULT Gaston, 1925, Métaphores occidentales - Essai sur les valeurs imaginatives concrètes du français parlé en Basse-Bretagne comparé avec les patois, parlers techniques et argots français, Paris : PUF.

ESNAULT Gaston, 1965, Dictionnaire historique des argots français, Paris : Larousse.

GOUDAILLIER Jean-Pierre, 1998 (1997), Comment tu tchatches ! Dictionnaire du français contemporain des cités, Paris : Maisonneuve et Larose. 
GREEN Jonathon, 2021, Green's Dictionary of Slang, digital edition, consulté le 15 novembre 2020, https://greensdictofslang.com GUIRAUD Pierre, 1980 (1956), L'argot, Paris : PUF, ‘Que Sais-Je ?'.

LAKOFF George \& JOHNSON Mark, 1985 (1980), Les métaphores dans la vie quotidienne (Metaphors We Live by), Paris : Les Éditions de Minuit.

LE GUERN Michel, 1973, Sémantique de la métaphore et de la métonymie, Paris : Larousse, http:// www.revue-texto.net/Parutions/LeGuern/LeGuern.html

MOURA Jean-Marc, 2019, " Jeux de mots, humour et traduction », in BRISSET Frédérique et al. (Eds.), Du jeu dans la langue, traduire le jeu de mots, Villeneuve d'Ascq : Presses Universitaires du Septentrion, 19-32.

POTTIER Bernard, 1964, « Vers une sémantique moderne », Travaux de linguistique et de littérature II : 107-137.

PRUVOST Jean \& SABLAYROLLES Jean-François, 2012 (2003), Les néologismes (2 $2^{\mathrm{e}}$ éd.), Paris : PUF, 'Que SaisJe?'.

SABLAYROLLES Jean-François, 1996, « Néologismes : une typologie des typologies », Cahiers du CIEL

(UFR EILA, Université Paris-VII) : 11-48. https://www.eila.univ-paris-diderot.fr/recherche/ clillac/ciel/cahiers/1996-1997

SABLAYROLLES Jean-François, 2006, « La néologie aujourd'hui », in GRUAZ Claude (Ed.), À la recherche du mot : De la langue au discours, Limoges : Lambert-Lucas, 141-157.

SABLAYROLLES Jean-François, 2012, « Des néologismes par détournement ? ou Plaidoyer pour la reconnaissance du détournement parmi les matrices lexicogéniques », in JULLION Marie-Christine, LONDEI Danielle \& PUCCINI Paola (Eds.), Recherches, didactiques, politiques linguistiques : perspectives pour l'enseignement du français en Italie, Milan : Francoangeli, 17-28.

SABLAYROLLES Jean-François, 2017, Les Néologismes. Créer des mots français aujourd'hui, « Les petits guides de la langue française - Le Monde ", $\mathrm{n}^{\circ}$ 29, Paris : Garnier.

SAUVAGE Laura, 2013, Stupidity in English Slang and Colloquial Language, Mémoire de master de Traduction et interprétation, Parcours MéLexTra, Université de Lille, sous la direction de F. Antoine.

SILBERSTEIN Zoë, 2020, Ten Pounds of Shit in a Five-Pound Society, or How Fatness Fails to Fit in: A Study of the Slang and Colloquialisms Used to Designate the Fat Individual in English, Mémoire de master de Traduction et interprétation, Parcours MéLexTra, Université de Lille, sous la direction de F. Antoine.

SUHAMY Henri, 1981, Les figures de style, Paris : PUF, 'Que Sais-Je?'

TOURNIER Jean, 1985, Introduction descriptive à la lexicogénétique de l'anglais contemporain, ParisGenève : Champion-Slatkine.

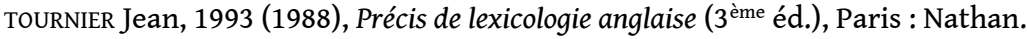

\section{NOTES}

1. Mémoire de recherche en première année de master (234) et mémoire de recherche appliquée en deuxième année (423). 
2. Je retiens le mot «formel» ici, pour mettre l'emprunt sur le même pied que, par exemple, la suffixation ou la composition, étant donné qu'il n'est pas morphologique.

3. On pourrait certainement adjoindre «bosser " à la série française : le Dictionnaire historique de la langue française Robert indique que le terme, d'abord argotique puis familier (1878) «s'explique peut-être comme une extension du sens de « se courber » attesté dans les dialectes de l'Ouest, par un développement « se courber sur son travail », d'où « travailler dur » (article « bosse »). Gaston Esnault, qui situe l'apparition du mot en $1878 \mathrm{chez}$ les maçons, avant diffusion à tous corps de métiers, en fait l'abrégé de "bosser du dos» (Rennes), signifiant donc "se courber sur son travail». Cependant, les sens techniques, dans plusieurs professions, peuvent expliquer, en accord avec la matrice évoquée pour la série repérée, ce sens générique de "travailler", notamment dans le vocabulaire de la marine («bosser une embarcation »: la retenir avec des bosses, qui sont des cordages ; le Petit Robert date de 1680, mais aussi « bosser un câble »).

4. J'utilise les appellations « sèmes centraux » et "sèmes périphériques », qui me semblent plus concrètes, et transparentes, pour ce que, par exemple, Tournier nomme «sèmes d'identification" et "sèmes accessoires" et que Bernard Pottier [1964:121-125] appelle « dénotatifs » et « connotatifs».

5. Les amples ensembles de mots réunis l'ont été principalement à partir de sources dictionnairiques (et de quelques articles dans certains cas), dont le nombre varie entre une quinzaine et une trentaine selon les champs et les mémoires.

6. 1948 est la date de publication d'une source secondaire. Il est évident que ces expressions, non répertoriées avant, sont plus anciennes.

7. L'information manque, malheureusement, ou reste très parcellaire, mais il serait naturellement intéressant ici de savoir si la création de chacun de ces items et aussi son usage est d'une femme ou d'un homme. Si l'on a peu de doute pour certains items, intuitivement, raisonnablement, attribuables à un homme (pour qui les règles rendent la femme |indisponible|, par exemple), même si l'on peut penser que beaucoup de ces mots et expressions sont «au prisme masculin», donc, il n'en reste pas moins que la différence entre « rire avec» et «rire contre » ou « de » ne peut se faire de façon indiscutable sans ces données.

8. Suhamy [1981:115] rappelle que l'antiphrase est le véhicule de l'ironie et du sarcasme : on est certes ici davantage dans le « rire contre » ou « de » que dans le « rire avec ».

9. Mais Esnault [1925:12] parlait déjà de «l'inspiration prise [par la métaphore] des choses à exprimer [...] la probable réaction de telle sorte selon les dates, les métiers... ».

10. Un détour par le dictionnaire de J. Green en ligne (ce qu'il n'était pas lors de la collecte du corpus auquel il est fait référence) livre d'autres expressions encore, par exemple a few bob short of the pound, a couple of chips short of a computer / a cookie / an order / a complete circuit / a casino bet / a butty, qui joue et rebondit sur divers sens de chip; a few spring rolls short of a banquet, a few French fries / three chips short of a Happy Meal etc. : la liste est très longue !

11. Le lexicographe risquera-t-il a few words short of a dictionary? Le bibliophile, a few books short of a library? Quelqu'un, a few fruits short of a salad? etc.

12. Mes italiques. 


\section{ABSTRACTS}

Building on the idea of a "word-creator", a lexical machine that combines semantic devices like metaphor and metonymy and formal devices (mainly borrowed from Tournier's lexicogenic devices) to produce new lexical items, a process that is especially observable in slang and colloquial language, both in English and in French, this paper focuses on series of synonymous slang and colloquial language words and expressions. Some of these are built around what we call a "semantic equation", or a semantic trait that is brought into sharp focus. Other sets of expressions, which are much larger, are built around the iteration and slight deformation of a prototypical expression that ends up being forgotten in the rather mechanical re-use of the expression's general outline. It is argued here that this mechanism illustrates the notion of slang as wordplay as the expressions thus created seem to echo one another over time and to build upon one another in a sort of attempt at being funnier, or more original or more absurd than those who came before.

Après avoir brièvement resitué le concept de matrices lexicogéniques (Tournier [1985], Sablayrolles [2012]), ou de « machine à créer du lexique » [Calvet 1994], en particulier pour ce qui concerne les lexiques argotiques et familiers, de l'anglais comme du français, on se penche ici sur un procédé en marge, ou plutôt une variante du croisement d'une des matrices formelles (qui sont en nombre fini) et d'une matrice sémantique (en nombre variable, en fonction des champs lexicaux, essentiellement métaphores ou métonymies), qui donne naissance à des séries d'items, mots ou expressions, synonymes en argot ou en langue familière. La production de telles séries peut se faire à partir d'une simple équation sémantique, sollicitée de façon répétée, ou de la mise en relief, les uns après les autres, de sèmes différents d'un même objet. Elle peut se faire aussi, et c'est là qu'elle se révèle la plus productive, grâce à l'itération, avec ou sans légère déformation, de la forme d'une expression prototypique reprise en écho au fil du temps, à l'envi : c'est là sans doute le procédé, que l'on nomme "rebond» ou "surenchère ", le plus humoristique de la création argotique et familière - étant bien entendu que l'humour est, naturellement, protéiforme -, qui mène à une sorte d'emballement de la machine à créer du lexique, avec des résultats souvent inattendus, voire loufoques.

\section{INDEX}

Mots-clés: argots, langue familière, machine à créer du lexique, séries lexicales, jeux de mots et avec les mots

Keywords: slang, colloquial language, lexicogenesis, lexical series, playing with and on words

\section{AUTHOR}

\section{FABRICE ANTOINE}

Université de Lille, ULR 4074 - CECILLE - Centre d'Études en Civilisations Langues et Lettres

Étrangères, Lille, France

fabrice.antoine@univ-lille.fr 